AperTO - Archivio Istituzionale Open Access dell'Università di Torino

\title{
Association between Beta1-Adrenergic Receptor Polymorphism and Risk of ICD Shock in Heart Failure Patients
}

This is a pre print version of the following article:

Original Citation:

Availability:

This version is available http://hdl.handle.net/2318/1562061

since 2020-02-28T15:37:43Z

Published version:

DOI:10.1111/pace.12860

Terms of use:

Open Access

Anyone can freely access the full text of works made available as "Open Access". Works made available under a Creative Commons license can be used according to the terms and conditions of said license. Use of all other works requires consent of the right holder (author or publisher) if not exempted from copyright protection by the applicable law. 


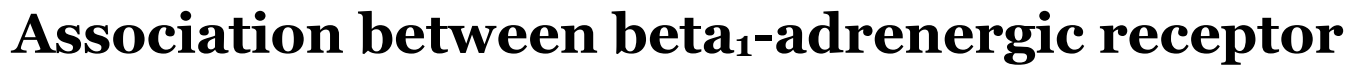 polymorphism and risk of ICD shock in heart failure patients}

5 Luisa ZANOLLA, MD*, Paola GUARISE, MD*, Luca TOMASI, MD*, Corrado VASSANELLI, MD*, Nicola CICORELLA, MD ${ }^{\dagger}$, Roberto ZANINI, MD ${ }^{\dagger}$, Simonetta GUARRERA, M.Sc. $¥ \S$, Giovanni FIORITO, M.Ed. Math $\S \ddagger$, Giuseppe MATULLO, M.Sc., $\mathrm{PhD} \neq \S$.

$10 *$ Azienda Ospedaliera Universitaria Integrata Verona - Dept.of Medicine - Cardiology Division - Piazzale Stefani 1 - Verona (Italy)

† Struttura Complessa di Cardiologia - Dipartimento Cardio-Toraco- Vascolare Azienda Ospedaliera "Carlo Poma" - Mantova (Italy)

₹ Dept. Medical Sciences - University of Torino (Italy)

$15 \S$ Human Genetics Foundation (HuGeF) - Via Nizza 52 - Torino (Italy)

Address for correspondence:

Luisa ZANOLLA

Dipartimento di Medicina - Sezione di Cardiologia

20 Piazzale Stefani 1 - 37126 Verona (Italy)

Tel.0039-45-8122040

Fax.0039-45-8122789

Mail: luisa.zanolla@univr.it

25 Short title $: \beta$ receptor SNPs and ICD shock 


\section{ABSTRACT}

\section{Background}

Sympathetic activation in heart failure patients favors the development of ventricular arrhythmias, thus leading to an increased risk of sudden cardiac death. $\beta_{1}$ and $\beta_{2}$ adrenergic receptor polymorphisms have been linked to the risk of sudden death. Implantable cardioverter-defibrillators (ICD) are implanted in a large percentage of heart failure patients, and beyond preventing sudden cardiac death they provide a continuous monitoring of major ventricular arrhythmias and of their own interventions. We investigated whether functionally relevant $\beta_{1}$ and $\beta_{2}$-adrenergic receptor polymorphisms are associated with risk of ICD shocks, as evidenced in ICD memory.

\section{Methods}

311 patients with systolic heart failure were enrolled, and number and timing of shocks in ICD memory were recorded.

Four selected polymorphisms were determined: $\beta_{1}$ adrenergic receptor polymorphisms Ser ${ }^{49}$ Gly and Arg389Gly and $\beta_{2}$ adrenergic receptor polymorphisms Arg ${ }^{16}$ Gly and Gln ${ }^{27 G l u . ~}$

\section{Results}

Only Ser49Gly was significantly correlated with time free from ICD shocks, both considering time to the first event in a Cox model (hazard ratio 2.117), and modeling repeated events with the Andersen-Gill method (hazard ratio 2.088). Gly allele 
50 carriers had a higher probability of ICD shock. The relationship remained significant even after adjusting for ejection fraction and beta-blocker dosage (hazard ratio 1.910).

\section{Conclusions}

Data from our study suggest that the $\beta$ adrenoreceptor Gly 49 allele of the $\beta_{1}$ adrenergic receptor Ser49Gly polymorphisms may increase the risk of ICD shock in patients with heart failure, independently of beta-blocker dosage. 


\section{Introduction}

In heart failure (HF) patients with reduced ejection fraction (EF), sympathetic activation, mediated by the $\beta_{1}$-adrenergic and $\beta_{2}$-adrenergic receptors, favors the development of ventricular arrhythmias, thus leading to an increased risk of sudden cardiac death. Implantable cardioverter-defibrillator (ICD) is therefore indicated in a large percentage of HF patients; beyond preventing sudden cardiac death, ICD provides a continuous monitoring of major ventricular arrhythmias and of its own interventions. ICD shocks represent an event with the peculiar characteristic that it can repeat several times.

In the present study, in order to assess the role of functionally relevant $\beta_{1}$ and $\beta_{2}$ adrenergic receptor polymorphisms, we investigated whether they are associated with the risk of ICD shocks.

Two $\beta_{1}$-adrenergic receptor functionally relevant single nucleotide

polymorphisms (SNPs) have been identified, with amino acid substitutions Arg389Gly (1) and Ser49Gly, and widely studied, associated with better prognosis in patients with HF in some, but not all, studies.

For the $\beta_{2}$-adrenergic receptor thirteen SNPs have been described; two common SNPs result in the amino acid substitutions Gly ${ }^{16} \mathrm{Arg}$ and Gln ${ }^{27} \mathrm{Glu}$. There are only a few reports suggesting a prognostic effect of these polymorphisms, both in general population (2) and in HF patients $(3,4)$, while there are not studies on their effect on ventricular arrhythmias.

The role of these four polymorphisms was investigated in the present study in 311 patients with HF, implanted with an ICD according to current guidelines (5). 


\section{Methods}

\section{Study population}

Consecutive eligible patients were enrolled from the HF outpatient clinic of two Hospitals (Verona and Mantova). Diagnosis of heart failure with reduced EF was based on criteria defined by European guidelines (5).

Eligibility criteria required that patients had to be implanted with ICD at least one month before, either for primary or for secondary prevention, according to current guidelines. Further eligibility criteria were age $>18$ years and reduced left ventricular $\mathrm{EF}$ at the time of ICD implantation, irrespective of functional class. All patients had to be of Caucasian ethnicity, as in the area non-Caucasian patients are very few, and their inclusion would have added heterogeneity, without reaching the statistical power to allow any comparison.

Patients with a documented history of myocardial infarction, percutaneous transluminal coronary angioplasty, coronary artery bypass graft or $>50 \%$ diameter stenosis of any of the major coronary epicardial arteries were classified as having ischemic HF. Other patients were classified as having nonischemic HF. Patients with HF caused by primary valvular disease, myocarditis, obstructive or hypertrophic 100 cardiomyopathies were excluded from the present evaluation.

Patients were followed by the outpatient HF clinic of the two Hospitals. All patients had to be on optimal medical therapy on enrolment, according to European guidelines (5).

The study was approved by the Institutional Review Board of both participating

105 hospitals; it was conducted in accordance with the Helsinki Declaration, and all patients provided written informed consent. 


\section{Demographic and clinical data}

Demographic variables included sex and age. Clinical variables included ICD

110 indication (primary vs. secondary prevention), the presence of right ventricular stimulation or biventricular stimulation, etiology (ischemic vs. nonischemic), and the presence of diabetes. These variables were obtained from medical records at baseline ICD assessment.

For any ICD intervention the dosage of $\beta$-blocker, titrated over time, was recorded; to keep into account different molecules, the dosage was expressed as the percentage of the target dosage in the European Guidelines (5). It was also recorded whether patient was on amiodarone, and if the rhythm before the shock was sinus rhythm or atrial fibrillation. For each ICD intervention, EF and diastolic filling pattern were recorded from the most recent routine echocardiogram, when available.

\section{ICD therapies}

All ICDs were programmed on an individual basis, without a standard protocol for the study. However each device had a "shock only" window for the treatment of high frequency ventricular arrhythmias ( $\geq 190 \mathrm{bpm})$. Arrhythmias in lower range

125 of frequencies were only monitored (in patients implanted in primary prevention), or treated in the first instance with Anti-Tachycardia Pacing (ATP) (in patients implanted in secondary prevention).

The study end-point was appropriate ICD shock, delivered either for ventricular tachycardia or ventricular fibrillation in the high frequency range. Patients were seen at the center of implantation at regular intervals. Each subject's ICD was interrogated during clinical follow-up visits. Arrhythmic events were recorded from the ICD and stored, or retrieved from archive storage. All therapy EGM recordings were reviewed by an expert electrophysiologist in order to rule out inappropriate ICD therapies. 
Therapies delivered to treat rhythm other than ventricular tachyarrhythmias (e.g. atrial fibrillation) were considered inappropriate. Only appropriate therapies were included in the analysis. It was recorded also if the ICD intervention took place during an electrical storm, defined as the occurrence of 3 or more shocks during a single 24 -h period (6).

The choice to include in the analysis only shocks and to exclude arrhythmias terminated by fast pacing, i.e. ATP, was based on considering that the time interval during which our data were collected was wide, and in the first years ATP was used mainly to treat slower tachycardias, with heart rate below 190-200; however, these arrhythmias do not always cause or proceed to cardiac arrest and cannot thus be considered a surrogate variable for fatal arrhythmias. Shock programming, on the contrary, was more homogenously applied over time in faster, not electrically organized arrhythmias, which are easily interpreted as a surrogate of sudden cardiac death $(7,8)$.

\section{Sample preparation and DNA Genotyping}

Blood samples were collected in ethylenediaminetetraacetic acid (EDTA) and stored frozen at $-80^{\circ} \mathrm{C}$ prior to DNA extraction. Genomic DNA was extracted from whole blood by an automated “on-column” DNA purification method on a QIAsymphony SP instrument (QIAGEN GmbH, Germany), according to manufacturer's protocols. DNA quality and concentration was assessed on a

155 NanoDrop 8000 spectrometer (ThermoScientific Inc.). A 5' nuclease assay with MGB TaqMan Probes (TaqMan® SNP Genotyping Assays, Life Technologies) on a ABI PRISM@ 7900HT Sequence Detection System instrument (Life Technologies) was used to genotype the 5 selected polymorphisms, namely ADRB1 rs1801252 (Ser49Gly), ADRB1 rs1801253 (Arg389Gly), ADRB2 rs1042713 (Arg' ${ }^{16}$ Gly), and ADRB2 rs1042714 
$160\left(\mathrm{Gln}{ }^{27} \mathrm{Glu}\right)$. Assay results were analyzed with the dedicated SDS software; all the automatic genotype calls were inspected by an operator to check for clusters quality and manually edited or removed when appropriate.

\section{Statistical analysis}

Continuous variables are presented as mean \pm standard deviation or median and interquartile range when a Gaussian distribution could not be assumed. Categorical variables are presented as absolute numbers and percentages. All statistical analyses were performed using Intercooled Stata version 8.0.

A Cox proportional hazard model was applied in order to determine the independent role of genetic polymorphisms as predictors of appropriate ICD shocks, both as univariate predictors and adjusting for covariates. The hazard ratio (HR) is reported along with its confidence interval (c.i.). The role of genetic polymorphisms was first assessed on all 3 genotypes, then analyzing heterozygotes in combination with homozygotes for the variant allele. The Andersen-Gill proportional-intensity

175 model (9) was used to identify the independent predictors of ICD shock. This technique allows all the events to be analyzed, in contrast to Cox modeling used in most studies, which only consider the first event. Coefficients are reported with their c.i. The model was applied using Intercooled Stata 8.o, for which the Andersen-Gill model algorithm had been published (10). 


\section{Results}

\section{Study population}

A total of 311 patients were enrolled in the study. Demographics are reported in Table 1.

All patients were Caucasians, born in Italy. Male subjects were 263 (84.6\%) and the average age was $64.2 \pm 10.6$ years. The etiology was ischemic in 188 patients (60.5\%). Sinus rhythm was observed in 221 patients (71.1\%). The ICD had been implanted in primary prevention in 244 patients (78.5\%); biventricular stimulation was applied in 210 patients (67.5\%). Median beta-blocker dosage on enrolment was $19050 \%$ (interquartile range 37.5-100).

Enrolment began on March 2009 and arrhythmia and ICD therapy endpoints were collected from the first record available (October 1998) up to November 2012. The median follow-up was 49.1 months (interquartile range 32.9-67.9).

\section{ICD therapies}

During follow-up 236 patients (75.9\%) did not experience any shock. The median follow-up of patients without ICD interventions was 42.9 months (interquartile range 29.2-64.7). In 75 patients, 284 shocks were documented. A single ICD shock was recorded in 33 patients (11\%). Multiple ICD shocks during follow-up were recorded in 42 patients. A total of 10 patients experienced 12 arrhythmic storms.

\section{Beta adrenergic receptor polymorphisms}

The genotype frequencies of the different polymorphisms are presented in Table 2. 
The genotype frequencies were in agreement with those predicted by the Hardy-Weinberg equilibrium.

\section{Beta adrenergic receptor polymorphisms and ICD shocks}

The univariate relationship at the Cox model between time free from ICD shock

210 and genetic polymorphisms is reported in Table 3. The relationship at the Cox model between time free from shock and homozygotes for the reference allele vs. carriers of the variant allele is reported in Table 4.

The analysis was then performed using the Andersen-Gill method, by which all events are kept into account. The results for ICD shock occurrence according to 215 genetic polymorphism are reported in Table 5 .

The influence of clinically relevant covariates was also assessed with the Andersen-Gill method: no effect was evident for gender, age, ICD indication (primary vs. secondary prevention), etiology (ischemic vs. nonischemic), or diabetes. The Andersen-Gill hazard ratio was significant for both $\beta$-blocker dosage (HR 0.976 for

220 each $1 \%$ increase, c.i. $0.966-0.988, p=0.0001)$ and for ejection fraction (HR 0.948 for each $1 \%$ increase; c.i. $0.910-0.988 ; p=0.011)$. No effect was evident for atrial fibrillation, amiodarone therapy, biventricular stimulation and mitral diastolic filling pattern.

When including in a multivariate model the $\beta_{1}$ Ser49Gly polymorphism, the only one related with ICD shock at univariate analysis, and the two significant covariates (namely beta-blocker dosage and ejection fraction), all of them maintained the statistical significance (Table 6), thus proving their independent predicting value.

An additional sensitivity analysis was conducted: the analysis for repeated ICD shocks was performed excluding ICD storms, in order to avoid an excessive influence of patients with several ICD shocks. Nevertheless, the significance of the effect of $\beta_{1}$ 
Ser49Gly remained, and it was further increased (HR 2.892; c.i. 1.631 - 5.129; $\mathrm{p}=0.0001]$ 


\section{Discussion}

Current guidelines (5) recommend ICD implantation for a significant proportion of the heart failure patients. It is however well known that, particularly in primary prevention, the proposed criteria have a low specificity. Even in our series, over a median 49 month period, $75 \%$ of the patients did not experience any ICD shock. Identifying new markers of arrhythmic risk could possibly improve risk stratification and ICD usage. Even potentially more interesting, on the opposite side, could be the identification of patients at higher risk of ICD shock, not only for the impact on the quality of life, but for the effect on long-term mortality of appropriate shocks, as evidenced by a recent meta-analysis (11).

Only in recent years an interest has arisen in the genetic influence on the risk of developing fatal ventricular arrhythmias in HF patients, in particular the presence of $\beta 1$ and $\beta 2$-adrenergic receptor polymorphisms.

For the $\beta_{1}$-adrenergic receptor two functionally relevant single nucleotide polymorphisms (SNPs) have been identified: a polymorphism leads to either a Glycine (Gly) or an Arginine (Arg) at amino acid position 389 (Arg389Gly) and another

250 polymorphism leads to either a Serine (Ser) or a Glycine (Gly) at amino acid position 49 (Ser49Gly). The Arg389 allele has demonstrated higher coupling affinity and hyperactive signaling in experimental heart failure models. It has been reported to be associated with congestive heart failure and ventricular tachycardia (1).

The $\beta_{1}$-adrenergic receptor polymorphism Arg389Gly was significantly related with

255 the presence of ventricular tachycardia on Holter monitoring, in one of the first papers on the topic, published by Iwai et al. (1) on 163 patients with idiopathic dilated cardiomyopathy; the Gly389 allele was associated with a lower frequency of ventricular tachycardia. In a paper by Biolo et al. (12) in a group of 201 patients with 
systolic HF of any etiology, the prevalence of non-sustained ventricular tachycardia, as

260 detected by Holter monitoring, was significantly affected by the $\beta_{1}$-adrenergic receptor polymorphism Arg389Gly, with a lower frequency in homozygous Gly389Gly patients; however, it was not affected by the Ser49Gly polymorphism. In a more recent paper by the same group (13), in seventy-three HF patients implanted with ICD, the time to the first appropriate ICD therapy was significantly shorter in carriers of two variant

265 alleles, defined as "risk" genotypes, namely Arg allele carriers of the $\beta_{1}$ Gly389Arg polymorphism and $\mathrm{T}$ allele carriers of the GNB3 $\mathrm{C} 25 \mathrm{~T}$ polymorphism, a gene coding for the $\mathrm{G}$ protein 3 subunit. When only the $\beta_{1} \mathrm{Gly}{ }^{389} \mathrm{Arg}$ polymorphism was considered, however, there was no statistically significant difference in appropriate ICD shocks in patients with at least one Arg389 allele, compared with Gly389Gly homozygous

270 patients. It should however be reminded that only 24 subjects that underwent therapies, considering both shock and ATP, were considered as appropriated and thus included in the analysis. Moreover, the use of a Cox regression model implies that only the first event of a patients is considered, while a median of 3 episodes per patient was recorded.

The use of the Andersen-Gill proportional-intensity regression model (9), an extension of the Cox proportional-hazards method, allows to take into account the risk of repeated events and not just the first event, thus increasing the statistical power of the design. Its use is becoming more common in recent years, and it has been applied in modeling the risk of recurrent syncope (14), ICD therapies (15) and hospital readmission in HF patients implanted with left ventricular assist device (16). With the use of this method we were able to outline that the Ser49Gly polymorphism of the $\beta_{1}$-adrenergic receptor significantly affects the risk of repeated ICD shocks, with the Gly49 allele carrying an increased risk of ICD shock. 
This result is at odds with the absence of effect of the Ser49Gly polymorphism on the prevalence of ventricular tachycardia reported by Biolo et al. (11). The difference in sensitivity between a 24-hour Holter monitoring and a prolonged follow-up through ICD memory must however be taken into account; moreover, 3 consecutive ventricular ectopic beats already define a non-sustained ventricular tachycardia, but their significance and their prognostic value is different from an arrhythmia inducing an appropriate ICD shock.

ICD shock could be considered as a surrogate for fatal arrhythmias and thus for sudden cardiac death, the latter being almost always caused by an arrhythmic event. Although this concept has been questioned, mainly for the observation that a reduction in ICD shock is not associated with an improvement in survival (17), one 295 could expect in any case a lower number of ICD shocks to be linked with a better prognosis and better quality of life (11).

The Gly49 allele, however, was associated in previous studies with a better prognosis, and this observation could indirectly conflict with our results. The most frequently quoted studies are two papers published by a Swedish group $(18,19)$; these

300 studies, however, enroll also patients with preserved ejection fraction, which have a different natural history; moreover in the first cohort (17) only $40 \%$ of the patients were on beta blockers. In the second cohort (18) with $83 \%$ of the patients on beta blockers, the five year transplant-free survival did not differ between Ser 49 homozygotes patients and Gly49 allele carriers.

The only other study to report a significant effect of Ser49Gly polymorphism is the one by Forleo et al. (3), which reports a better 33 month transplant and hospitalization-free survival in Ser49Gly heterozygous patients compared to Ser 49 homozygous patients. In synthesis two studies, both on idiopathic dilated 
cardiomyopathy patients only, describe a significant protective effect of the Gly49 310 allele.

A series of other studies, however, did not detect any prognostic effect of Ser49Gly polymorphism; all these studies were conducted on patients with heart failure of any etiology. No influence on all-cause mortality and heart failure-related mortality was found by Biolo et al. (11) in 201 patients; no result on transplant-free survival was found by de Groote et al. (20) in 444 patients, by Shin et al.(4) in 227 patients, by Sehnert et al. (21) in 637 patients and by Leineweber et al. (22) in 226 end-stage HF patients. The absence of a prognostic influence of Ser49Gly polymorphism is confirmed by the meta-analysis by Liu et al. (23). Overall mortality was not affected; the result was probably mainly driven by the study by Wang et al. (24), which, opposite to the previously mentioned studies, documented a lower heart-failure related mortality in Ser 49 homozygotes in a population of 430 Chinese patients, but none of the three studies included in the meta-analysis had a better prognosis for Gly49 allele carriers. A larger number of studies was included for the composite end-point of death, hospitalization and transplant, but the result was always not significant. In vivo results 325 are conflicting on the role of Ser ${ }^{49}$ Gly polymorphism on prognosis, and thus do not contradict our result on the Gly49 allele being associated with an increased risk of ICD shock.

Another possible conflict between our results and published data is the absence of any effect of the Arg ${ }^{389}$ Gly polymorphism on time free from ICD shock. As already

330 pointed out, the only paper examining the influence of $\beta$-adrenoreceptor polymorphisms on ICD interventions (12) did not show any significant effect of Arg389Gly polymorphism when considered alone. As far as prognosis is concerned, in the paper by Biolo et al. (11) HF-related mortality was significantly reduced in Gly389Gly patients. Other papers, however, failed to identify any prognostic effect of 

(25) on 600 patients, and in the study of de Groote et al. (19) on 444 patients, no effect on hospitalization-free survival was documented. In the paper by Sehnert et al. (20) no effect on transplant-free survival was evident in 637 patients on beta blocker treatment. In the paper by Forleo et al. (3) no effect on hospitalization and transplant340 free survival was evident, whereas in the study by Leineweber et al. (21) in 226 endstage HF patients no prognostic effect was reported. So even prognosis, an end-point which obviously does not coincide with time free from ICD shocks, does not have a definite relationship with the Arg389Gly polymorphism.

For the $\beta_{2}$-adrenergic receptor thirteen SNPs have been described; two common SNPs result in the amino acid substitutions Gly ${ }^{16} \mathrm{Arg}$ and $\mathrm{Gln}{ }^{27} \mathrm{Glu}$. These two variants are in strong linkage disequilibrium; Glu27 almost always is paired with Gly16 in humans. In an epidemiological study Gln27 homozygous individuals have evidenced an increased risk of sudden cardiac death in two different populations without HF (2). In another study on HF patients (3), the presence of the Arg16 allele and the

350 homozigosity Gln ${ }^{27}$ Gln were associated with a better prognosis in patients with idiopathic dilated cardiomyopathy, but only the simultaneous presence of two copies of Arg16 Gln27 was associated with a worse prognosis in another study (4), in patients with $\mathrm{HF}$ of all etiologies. However, no prognostic effect in HF patients was found in other studies $(19,20,21)$. We were not able to identify any effect of these $\beta_{2}$-adrenergic receptor polymorphism.

\section{Conclusions}

In conclusion, data from our study suggest that the Gly 49 allele of the $\beta_{1}$-adrenergic receptor Ser49Gly polymorphisms may identify patients with heart failure at increased risk of ICD shock and thus of life-threatening arrhythmias. 
The main drawback of the current study is the limited number of patients; the hypothesis ought to be verified in a larger study, which could also assess the role of gene haplotypes. 


\section{Author contributions:}

\section{Luisa ZANOLLA}

Took part in:

- conception and design of the study

- substantial contributions to the acquisition of data

- analysis and interpretation of data

- statistical analysis

- drafting of the manuscript

- approval of the manuscript submitted

\section{Paola GUARISE}

Took part in:

- conception and design of the study

- substantial contributions to the acquisition of data

- interpretation of data

- drafting of the manuscript

- critical revision of the manuscript

- approval of the manuscript submitted

\section{Luca TOMASI}

Took part in:

- conception and design of the study

- substantial contributions to the acquisition of data

- interpretation of data

- drafting of the manuscript

- approval of the manuscript submitted

\section{Corrado VASSANELLI}

Took part in:

- substantial contributions to research design

- interpretation of data

- critical revision of the manuscript 
- approval of the manuscript submitted

395 Nicola CICORELLA

Took part in:

- conception and design of the study

- substantial contributions to the acquisition of data

- interpretation of data

400

- critical revision of the manuscript

- approval of the manuscript submitted

\section{Roberto ZANINI}

Took part in:

- substantial contributions to research design

405

- interpretation of data

- critical revision of the manuscript

- approval of the manuscript submitted

\section{Simonetta GUARRERA}

Took part in:

410

- conception and design

- substantial contributions to the acquisition of data

- analysis and interpretation of data

- approval of the manuscript submitted

\section{Giovanni FIORITO}

Took part in:

- conception and design of the study

- substantial contributions to the acquisition of data

- analysis and interpretation of data

- approval of the manuscript submitted

\section{Giuseppe MATULLO}

Took part in:

- conception and design of the study

- substantial contributions to the acquisition of data 
- analysis and interpretation of data

425

- drafting of the manuscript

- critical revision of the manuscript

- approval of the manuscript submitted 


\section{References}

1. Iwai C, Akita H, Shiga N, Takai E, Miyamoto Y, Shimizu M, Kawai H, et al. Suppressive effect of the Gly389 allele of the beta1-adrenergic receptor gene on the occurrence of ventricular tachycardia in dilated cardiomyopathy. Circ $J$ 2002;66:723-8.

2. Sotoodehnia N, Siscovick DS, Vatta M, Psaty BM, Tracy RP, Towbin JA, Lemaitre $\mathrm{RN}$, et al. Beta2-adrenergic receptor genetic variants and risk of sudden cardiac death. Circulation 2006;113:1842-8.

3. Forleo C, Resta N, Sorrentino S, Guida P, Manghisi A, De Luca V, Romito R, et al. Association of beta-adrenergic receptor polymorphisms and progression to heart failure in patients with idiopathic dilated cardiomyopathy. Am J Med 2004;117:451-8.

4. Shin J, Lobmeyer MT, Gong Y, Zineh I, Langaee TY, Yarandi H, Schofield RS, Et al. Relation of beta(2)-adrenoceptor haplotype to risk of death and heart transplantation in patients with heart failure. Am J Cardiol 2007;99:250-5.

5. McMurray JJ, Adamopoulos S, Anker SD, Auricchio A, Böhm M, Dickstein K, Falk V, et al. ESC Guidelines for the diagnosis and treatment of acute and chronic heart failure 2012: The Task Force for the Diagnosis and Treatment of Acute and Chronic Heart Failure 2012 of the European Society of Cardiology. Developed in collaboration with the Heart Failure Association (HFA) of the ESC. Eur Heart J 2012;33:1787-847. 
6. Credner SC, Klingenheben T, Mauss O, Sticherling C, Hohnloser SH. Electrical storm in patients with transvenous implantable cardioverter-defibrillators. Incidence, management and prognostic implications. J Am Coll Cardiol 1998;32:1909-15.

7. Wilkoff BL, Williamson BD, Stern RS, Moore SL, Lu F, Lee SW, BirgersdotterGreen UM, et al.; PREPARE Study Investigators. Strategic programming of detection and therapy parameters in implantable cardioverter-defibrillators reduces shocks in primary prevention patients: results from the PREPARE (Primary Prevention Parameters Evaluation) study. J Am Coll Cardiol 2008;52:541-50.

8. Madhavan M, Friedman PA. Optimal programming of implantable cardiacdefibrillators. Circulation 2013;128:659-72.

9. Andersen PK, Gill RD. Cox's regression model for counting processes: a large sample study. Ann Stat 1982;10:1100-1120.

10. Cleves M. Analysis of multiple failure-time data with Stata. Stata Technical Bulletin 49 .

11. Proietti R, Labos C, Davis M, Thanassoulis G, Santangeli P, Russo V, Di Biase L, et al. A systematic review and meta-analysis of the association between implantable cardioverter-defibrillator shocks and long-term mortality. Can J Cardiol 2015;31:270-7. 
12. Biolo A, Clausell N, Santos KG, Salvaro R, Ashton-Prolla P, Borges A, Rohde LE. Impact of beta1-adrenergic receptor polymorphisms on susceptibility to heart failure, arrhythmogenesis, prognosis, and response to beta-blocker therapy. Am J Cardiol 2008;102:726-32.

13. Chemello D, Rohde LE, Santos KG, Silvello D, Goldraich L, Pimentel M, Rosa PR, et al. Genetic polymorphisms of the adrenergic system and implantable cardioverter-defibrillator therapies in patients with heart failure. Europace 2010;12:686-91.

14. Liu JF, Moss AJ, Jons C, Benhorin J, Schwartz PJ, Spazzolini C, Crotti L, et al. Mutation-specific risk in two genetic forms of type 3 long QT syndrome. Am $J$ Cardiol 2010;105:210-3.

15. Ouellet G, Huang DT, Moss AJ, Hall WJ, Barsheshet A, McNitt S, Klein H, et al. Effect of cardiac resynchronization therapy on the risk of first and recurrent ventricular tachyarrhythmic events in MADIT-CRT. J Am Coll Cardiol 2012;60:1809-16.

16. Hasin T, Marmor Y, Kremers W, Topilsky Y, Severson CJ, Schirger JA, Boilson BA, et al. Readmissions after implantation of axial flow left ventricular assist device. J Am Coll Cardiol 2013;61:153-63.

17. Ha AH, Ham I, Nair GM, Connolly SJ, Dorian P, Morillo CA, Healey JS. Implantable cardioverter-defibrillator shock prevention does not reduce mortality: a systemic review. Heart Rhythm 2012;9:2068-74. 
18. Börjesson M, Magnusson Y, Hjalmarson AR, Andersson B. A novel polymorphism in the gene coding for the beta1-adrenergic receptor associated with survival in patients with heart failure. Eur Heart $J$ 2000;21:1853-1858.

19. Magnusson Y, Levin MC, Eggertsen R, Nyström E, Mobini R, Schaufelberger M, Andersson B. Ser49Gly of beta1-adrenergic receptor is associated with effective beta-blocker dose in dilated cardiomyopathy. Clin Pharmacol Ther 2005;78:221-31.

20.de Groote P, Lamblin N, Helbecque N, Mouquet F, Mc Fadden E, Hermant X, Amouyel P, et al. The impact of beta-adrenoreceptor gene polymorphisms on survival in patients with congestive heart failure. Eur J Heart Fail 2005;7:96673 .

21. Sehnert AJ, Daniels SE, Elashoff M, Wingrove JA, Burrow CR, Horne B, Muhlestein JB, et al. Lack of association between adrenergic receptor genotypes and survival in heart failure patients treated with carvedilol or metoprolol. $J$ Am Coll Cardiol 2008;52:644-51.

22. Leineweber K, Frey UH, Tenderich G, Toliat MR, Zittermann A, Nürnberg P, Körfer R, et al. The Arg16Gly- $\beta(2)$-adrenoceptor single nucleotide polymorphism: exercise capacity and survival in patients with end-stage heart failure. Naunyn Schmiedebergs Arch Pharmacol 2010;382:357-65.

23. Liu WN, Fu KL, Gao HY, Shang YY, Wang ZH, Jiang GH, Zhang Y, et al. $\beta 1$ adrenergic receptor polymorphisms and heart failure: a meta-analysis on 
susceptibility, response to $\beta$-blocker therapy and prognosis. PLoS One 2012;7:e37659. doi: 10.1371/journal.pone.0037659.

24. Wang L, Lu L, Zhang F, Chen Q, Shen W. Polymorphisms of beta-adrenoceptor and natriuretic peptide receptor genes influence the susceptibility to and the severity of idiopathic dilated cardiomyopathy in a Chinese cohort. J Card Fail 2010;16:36-44.

25. White HL, de Boer RA, Maqbool A, Greenwood D, van Veldhuisen DJ, Cuthbert R, Ball SG, et al; MERIT-HF Study Group. An evaluation of the beta-1 adrenergic receptor Arg389Gly polymorphism in individuals with heart failure: a MERIT-HF sub-study. Eur J Heart Fail 2003;5:463-8 